\title{
Análise documental de aspectos relacionados à prevalência, prevenção, assistência e complicações do diabetes em adultos das unidades federativas brasileiras
}

\author{
Documentary analysis of aspects related to the prevalence, prevention, care and complications of \\ diabetes in adults from Brazilian federative units \\ Análisis documental de aspectos relacionados con la prevalencia, prevención, atención y \\ complicaciones de la diabetes en adultos de unidades federativas brasileñas
}

Recebido: 08/04/2021 | Revisado: 14/04/2021 | Aceito: 16/04/2021 | Publicado: 01/05/2021

\author{
Renan Akira Fujii de Oliveira \\ ORCID: https://orcid.org/0000-0003-1479-0413 \\ Universidade Estadual Paulista, Brasil \\ E-mail: rakirafujii@gmail.com \\ Suzely Adas Saliba Moimaz \\ ORCID: https://orcid.org/0000-0002-4949-529X \\ Universidade Estadual Paulista, Brasil \\ E-mail: suzely.moimaz@unesp.br \\ Cléa Adas Saliba Garbin \\ ORCID: https://orcid.org/0000-0001-5069-8812 \\ Universidade Estadual Paulista, Brasil \\ E-mail: clea.saliba-garbin@unesp.br \\ Artênio José Isper Garbin \\ ORCID: https://orcid.org/0000-0002-7017-8942 \\ Universidade Estadual Paulista, Brasil \\ E-mail: artenio.garbin@unesp.br \\ Fernando Yamamoto Chiba \\ ORCID: https://orcid.org/0000-0003-4406-405X \\ Universidade Estadual Paulista, Brasil \\ E-mail: fernando.chiba@unesp.br
}

\begin{abstract}
Resumo
O diabetes é uma doença crônica que representa severo problema de saúde pública. Objetivou-se avaliar aspectos relacionados à prevalência, prevenção, assistência e complicações do diabetes em adultos nas unidades federativas (UF) brasileiras. Trata-se de um estudo ecológico, documental, realizado com dados da Pesquisa Nacional de Saúde2013 e 2019, coletados na página eletrônica do Instituto Brasileiro de Geografia e Estatística. A análise dos dados foi realizada para verificar a diferença nas proporções de indivíduos, segundo as variáveis investigadas, entre 2013 e 2019. Houve aumento na prevalência de diabetes em 26 UF (96,30\%), com variação de 3,3(9,3\%); e redução, em todas as UF, na proporção que nunca avaliou a glicemia, com variação de 3,6(25,9\%). Elevou-se a proporção de pessoas que utilizaram medicamentos para controle da doença em todas as UF, com variação de 48,1(93,7\%), entretanto, apenas 5 UF $(18,52 \%)$ apresentaram aumento na proporção que obteve medicamento pelo programa "Farmácia Popular". Aumentou a proporção de pessoas que receberam assistência médica no último ano em 22 UF $(81,48 \%)$, com variação de $53,2(87,3 \%)$, contudo, na maioria das UF diminuiu a proporção que realizou todos os exames complementares $(n=17)$ e todas as consultas com médico especialista $(n=18)$. Em 14 UF $(51,85 \%)$ elevou-se a proporção de internações, com variação de $4,7(31,4 \%)$, enquanto a proporção de pessoas com limitações nas atividades habituais diminuiu em 18UF (66,67\%), com variação de 2,4(20,5\%). A prevalência da doença aumentou na ampla maioria das UF, evidenciando a necessidade de estratégias de prevenção, assistência médica e farmacêutica, visando reduzir os agravos causados pela doença.
\end{abstract}

Palavras-chave: Diabetes Mellitus; Saúde pública; Assistência à saúde; Prevenção de doenças.

\begin{abstract}
Diabetes is a chronic disease that represents a severe public health problem. The objective was to evaluate aspects related to the prevalence, prevention, care and complications of diabetes in adults in Brazilian federative units(FU). This is an ecological, documentary study, performed with data from the National Health Survey-2013 and 2019, collected from website of the Brazilian Institute of Geography and Statistics. Data analysis was performed to verify the difference in proportions, according to the investigated variables, between 2013 and 2019. There was an increase in the prevalence of diabetes in $26 \mathrm{FU}(96.30 \%)$, varying 3.3(9.3\%); and reduction, in all $\mathrm{FU}$, in the proportion that never evaluated glycemia, varying 3.6(25.9\%). The proportion of people who used drugs to control the disease in all
\end{abstract}


FU increased, varying 48.1(93.7\%), however, only 5 FU(18.52\%) showed an increase in the proportion who obtained medication at "Farmácia Popular" program. The proportion of people who received medical assistance in the last year increased by $22 \mathrm{FU}(81.48 \%)$, varying 53.2(87.3\%), however, in the majority of FU the proportion that performed all complementary exams decreased $(n=17)$ and all consultations with a specialist doctor $(n=18)$. In 14 FU(51.85\%) the proportion of hospitalizations increased, varying $4.7(31.4 \%)$, while the proportion of people with limitations in usual activities decreased by $18 \mathrm{FU}(66.67 \%)$, varying $2.4(20.5 \%)$. The prevalence of the disease has increased in the vast majority of FU, highlighting the need for prevention strategies, medical and pharmaceutical assistance, aiming to reduce the problems caused by the disease.

Keywords: Diabetes Mellitus; Public health; Delivery of health care; Disease prevention.

\section{Resumen}

La diabetes es una enfermedad crónica que representa un grave problema de salud pública. El objetivo fue evaluar aspectos relacionados con la prevalencia, prevención, atención y complicaciones de la diabetes en adultos en unidades federativas brasileñas (UF). Se trata de estudio estudio ecológico documental, realizado con datos de la Encuesta Nacional de Salud-2013 y 2019, recopilados del sitio web del Instituto Brasileño de Geografía y Estadística. Se realizó un análisis de datos para verificar la diferencia de proporciones, según las variables investigadas, entre 2013 y 2019. Hubo un aumento en la prevalencia de diabetes en 26UF (96,30\%), variando 3,3(9,3\%); y reducción, en todas las UF, de la proporción que nunca evaluó glucemia, variando 3,6(25,9\%). La proporción de personas que consumían medicamentos para la enfermedad en todas las UF aumentó, variando 48,1 (93,7\%), sin embargo, solo 5UF(18,52\%) mostraron aumento en la proporción que obtuvieron medicación en el programa "Farmácia Popular". La proporción de personas que recibieron asistencia médica en el último año aumentó en $22 \mathrm{UF}(81,48 \%)$, variando 53,2(87,3\%), sin embargo, en la mayoría de UF la proporción que realizó todos los exámenes complementarios disminuyó(n=17) y todos consultas con médico especialista $(\mathrm{n}=18)$. En $14 \mathrm{UF}(51,85 \%)$ la proporción de hospitalizaciones aumentó, variando 4,7(31,4\%), mientras que la proporción de personas con limitaciones en las actividades habituales disminuyó en $18 \mathrm{UF}(66,67 \%)$, variando 2,4(20,5\%). La prevalencia de la enfermedad ha aumentado en la gran mayoría de las UF, destacando la necesidad de estrategias de prevención, asistencia médica y farmacéutica, con el objetivo de reducir los problemas provocados por la enfermedad.

Palabras clave: Diabetes Mellitus; Salud pública; Prestación de atención de salud; Prevención de enfermedades.

\section{Introdução}

O diabetes mellitus é uma condição de saúde crônica que compreende um grupo de distúrbios metabólicos caracterizados pela presença de hiperglicemia na ausência de tratamento, cuja etiopatologia heterogênea inclui deficiência na produção, secreção e/ou ação da insulina, e distúrbios do metabolismo de carboidratos, lipídios e proteínas (Leslie et al., 2016). Os principais tipos de diabetes mellitus são o diabetes mellitus tipo 1 e o diabetes mellitus tipo 2 . O diabetes mellitus tipo 1 é caracterizado pela destruição autoimune das células $\beta$ pancreáticas e deficiência na secreção de insulina, manifestando-se com maior frequência na infância e no início da idade adulta (DiMeglio, Evans-Molina, \& Oram, 2018). O diabetes mellitus tipo 2 é o mais comum e caracteriza-se por variados graus de disfunção das células $\beta$ pancreáticas e resistência à insulina, manifestando-se com maior frequência em adultos e idosos, entretanto, tem sido observado aumento expressivo em crianças e adolescentes, refletindo o crescente problema da obesidade infantil (Kao \& Sabin, 2016). Recentemente, em função dos avanços no conhecimento das vias fisiopatológicas, tecnologias para detecção da doença e tratamentos que atuam em vias específicas, a Organização Mundial da Saúde tem proposto novas classificações para o diabetes, incluindo formas híbridas de diabetes, diabetes não classificado, hiperglicemia detectada pela primeira vez durante a gravidez, e outros tipos específicos, visando orientar as decisões de cuidados clínicos, estimular a pesquisa em etiopatologia e fornecer uma base para realização de estudos epidemiológicos (World Health Organization, 2019).

O diabetes é um severo problema de saúde pública em todo o mundo. As estimativas globais atuais indicam que esta condição afetou 463 milhões de pessoas em 2019 e deve aumentar para 700 milhões até o ano de 2045. O Brasil ocupa a quinta posição na classificação dos países que possuem o maior número de adultos, com idade entre 20 e 79 anos, portadores de diabetes diagnosticados, apresentando 16,8 milhões de indivíduos acometidos em 2019 e estimativa de 26 milhões até o ano de 2045, representando um aumento de 55\% na prevalência da doença (International Diabetes Federation, 2019).

Os sintomas mais comuns da doença são a polidipsia, poliúria, visão turva e a perda de peso, entretanto, deve-se 
considerar que estas alterações podem não estar presentes em alguns casos ou manifestar-se de maneira pouco acentuada, contribuindo para a ausência e/ou retardo do diagnóstico e da busca por tratamento adequado (Skyler et al., 2017; Al-Lawati, 2017). Pessoas que vivem com diabetes possuem o risco de desenvolver uma série de complicações graves e potencialmente fatais, resultando em uma maior necessidade de cuidados médicos, qualidade de vida reduzida e grande carga de estresse nas famílias (Jing et al., 2018). As consequências em longo prazo do diabetes, quando não-tratado/controlado adequadamente, incluem retinopatia, nefropatia, neuropatia, doenças cardiovasculares, doença arterial periférica e cerebrovascular, obesidade, catarata, disfunção erétil, doença do fígado gorduroso não-alcoólica, e risco aumentado para o surgimento de infecções (Papatheodorou et al., 2018).

Os efeitos do diabetes e suas complicações vão além do acometimento da saúde do indivíduo, comprometendo famílias e sociedades inteiras. A doença tem amplas consequências socioeconômicas, impactando os sistemas de saúde e a economia, especialmente em países de baixa e média renda, nos quais o diabetes costuma ser acompanhado de outras doenças, evidenciando a importância de estratégias de prevenção e monitoramento (International Diabetes Federation, 2019).

No Brasil, o Programa HIPERDIA foi desenvolvido como parte do Plano de reorganização da atenção à hipertensão arterial e ao diabetes mellitus e destina-se ao cadastramento e acompanhamento de portadores destas enfermidades atendidos na rede ambulatorial do Sistema Único de Saúde - SUS, servindo como orientação para os gestores públicos na adoção de estratégias de intervenção (Brasil, 2001).

Diante da crescente prevalência da doença e da severidade de suas consequências e complicações, bem como da contínua necessidade de avaliação da condição de saúde da população, objetivou-se avaliar aspectos relacionados à prevalência, prevenção, assistência e complicações do diabetes em adultos nas unidades federativas (UF) brasileiras.

\section{Metodologia}

Trata-se de um estudo documental, ecológico, com análise temporal, realizado a partir dos dados da Pesquisa Nacional de Saúde de 2013 e 2019. Foram coletados dados sobre a prevalência do diabetes e aspectos relacionados à prevenção, assistência médica e farmacêutica, além de complicações da doença em indivíduos com 18 anos ou mais, segundo as unidades federativas (UF) brasileiras, por meio de consulta ao banco de dados da Pesquisa Nacional de Saúde de 2013 e 2019, disponível na página eletrônica do Instituto Brasileiro de Geografia e Estatística (Brasil 2014; Brasil, 2020). Considerando a crescente prevalência global da diabetes, a comparação dos resultados dessas pesquisas foi realizada para compreender a evolução das variáveis estudadas nas unidades federativas (UF) brasileiras.

Foram analisadas as seguintes variáveis: Proporção de pessoas (\%) que referem diagnóstico médico de diabetes; proporção de pessoas (\%) que nunca fizeram exame de sangue para medir a glicemia; proporção de pessoas (\%) que referem diagnóstico médico de diabetes e tomaram medicamento para diabetes ou usaram insulina nas duas últimas semanas anteriores à data da pesquisa; proporção de pessoas (\%) que tomaram algum medicamento para controlar a diabetes e obtiveram pelo menos um medicamento no programa "Farmácia Popular"; proporção de pessoas (\%) que referem diagnóstico médico de diabetes e receberam assistência médica para diabetes há menos de 1 ano; proporção de pessoas (\%) que referem diagnóstico médico de diabetes e que realizaram a última consulta em unidade básica de saúde; proporção de pessoas (\%) que referem diagnóstico médico de diabetes, e conseguiram realizar todos os exames complementares solicitados; proporção de pessoas (\%) que referem diagnóstico médico de diabetes e conseguiram realizar todas as consultas com médico especialista para as quais foram encaminhadas; proporção de pessoas (\%) que referem diagnóstico médico de diabetes e realizaram exame de vista há menos de 1 ano; proporção de pessoas (\%) que referem diagnóstico médico de diabetes e tiveram seus pés examinados há menos de 1 ano; proporção de pessoas (\%) que referem diagnóstico médico de diabetes e se internaram por causa da diabetes ou de alguma complicação da doença; e proporção de pessoas (\%) que referem diagnóstico médico de diabetes e possuem grau 
intenso ou muito intenso de limitações nas atividades habituais devido à diabetes ou de alguma complicação da doença.

A análise dos dados foi realizada para verificar a diferença nas proporções de indivíduos, segundo as variáveis investigadas, entre 2013 e 2019, de modo que diferenças com valores positivos indicam aumento na proporção, enquanto valores negativos indicam diminuição na proporção. Os dados foram analisados utilizando técnicas de estatística descritiva e os resultados apresentados sob a forma de tabelas.

Essa pesquisa utilizou dados secundários disponíveis no endereço eletrônico oficial do Instituto Brasileiro de Geografia e Estatística, sem qualquer tipo de identificação dos participantes, sendo dispensada de apreciação em comitê de ética em pesquisa, em conformidade com a Resolução no 466/2012 do Conselho Nacional de Saúde.

\section{Resultados}

A Pesquisa Nacional de Saúde trata-se de um inquérito de base populacional, representativo da população brasileira residente em domicílios particulares construídos com a finalidade exclusiva de habitação. As amostras para a sua realização, nos anos de 2013 e 2019, foram compostas por 81.767 e 108.525 domicílios, respectivamente.

Conforme demonstrado na Tabela 1, verificou-se que, entre os anos de 2013 e 2019, houve aumento na proporção de pessoas, com 18 anos ou mais, que referem diagnóstico médico de diabetes em todas as UF, com variação de 3,3 a 9,3\%, com exceção do estado do Amapá, que apresentou discreta redução (-0,70\%). Esta mudança foi acompanhada de redução, em todas as UF, na proporção de indivíduos que nunca avaliaram a glicemia com variação de 3,6 a 25,9\%. A região sudeste apresentou a menor proporção de pessoas que nunca realizaram o exame. 
Tabela 1. Evolução da proporção de pessoas (\%) com 18 anos ou mais, que referem diagnóstico médico de diabetes e da proporção de pessoas (\%) que nunca fizeram exame de sangue para medir a glicemia, segundo as unidades federativas brasileiras, em 2013 e 2019.

\begin{tabular}{|c|c|c|c|c|c|c|}
\hline \multirow[t]{2}{*}{ Unidades federativas } & \multicolumn{3}{|c|}{$\begin{array}{l}\text { Pessoas que referem diagnóstico médico de } \\
\text { diabetes }(\%)\end{array}$} & \multicolumn{3}{|c|}{$\begin{array}{l}\text { Pessoas que nunca fizeram exame de sangue } \\
\text { para medir a glicemia (\%) }\end{array}$} \\
\hline & 2013 & 2019 & Diferença & 2013 & 2019 & Diferença \\
\hline \multicolumn{7}{|l|}{ Região Norte } \\
\hline Rondônia & 5,0 & 5,3 & 0,30 & 16,4 & 12,1 & $-4,30$ \\
\hline Acre & 3,3 & 4,3 & 1,00 & 24,7 & 19,4 & $-5,30$ \\
\hline Amazonas & 4,6 & 5,4 & 0,80 & 15,6 & 11,4 & $-4,20$ \\
\hline Roraima & 4,0 & 5,0 & 1,00 & 22,7 & 9,8 & $-12,90$ \\
\hline Pará & 3,8 & 5,6 & 1,80 & 19,1 & 11,5 & $-7,60$ \\
\hline Amapá & 5,0 & 4,3 & $-0,70$ & 15,9 & 7,4 & $-8,50$ \\
\hline Tocantins & 5,4 & 6,3 & 0,90 & 19,1 & 10,6 & $-8,50$ \\
\hline \multicolumn{7}{|l|}{ Região Nordeste } \\
\hline Maranhão & 5,4 & 5,5 & 0,10 & 25,9 & 12,0 & $-13,90$ \\
\hline Piauí & 5,0 & 6,8 & 1,80 & 17,8 & 6,4 & $-11,40$ \\
\hline Ceará & 4,9 & 8,5 & 3,60 & 20,9 & 12,1 & $-8,80$ \\
\hline Rio Grande do Norte & 5,6 & 8,8 & 3,20 & 10,0 & 6,1 & $-3,90$ \\
\hline Paraíba & 4,5 & 7,5 & 3,00 & 9,9 & 7,7 & $-2,20$ \\
\hline Pernambuco & 6,2 & 7,1 & 0,90 & 11,2 & 7,0 & $-4,20$ \\
\hline Alagoas & 6,8 & 7,8 & 1,00 & 13,0 & 7,2 & $-5,80$ \\
\hline Sergipe & 6,0 & 6,8 & 0,80 & 8,6 & 4,3 & $-4,30$ \\
\hline Bahia & 5,0 & 6,7 & 1,70 & 11,1 & 8,8 & $-2,30$ \\
\hline \multicolumn{7}{|l|}{ Região Sudeste } \\
\hline Minas Gerais & 6,4 & 8,0 & 1,60 & 11,3 & 3,8 & $-7,50$ \\
\hline Espírito Santo & 6,1 & 6,8 & 0,70 & 6,7 & 3,6 & $-3,10$ \\
\hline Rio de Janeiro & 6,4 & 9,3 & 2,90 & 5,2 & 3,6 & $-1,60$ \\
\hline São Paulo & 7,7 & 8,6 & 0,90 & 7,4 & 4,3 & $-3,10$ \\
\hline \multicolumn{7}{|l|}{ Região Sul } \\
\hline Paraná & 5,7 & 7,7 & 2,00 & 12,4 & 5,4 & $-7,00$ \\
\hline Santa Catarina & 5,5 & 6,9 & 1,40 & 12,6 & 5,9 & $-6,70$ \\
\hline Rio Grande do Sul & 7,0 & 8,8 & 1,80 & 12,1 & 5,0 & $-7,10$ \\
\hline \multicolumn{7}{|l|}{ Região Centro-Oeste } \\
\hline Mato Grosso do Sul & 7,8 & 7,8 & 0,00 & 14,2 & 5,7 & $-8,50$ \\
\hline Mato Grosso & 6,2 & 6,6 & 0,40 & 17,6 & 7,8 & $-9,80$ \\
\hline Goiás & 6,4 & 7,4 & 1,00 & 14,3 & 6,0 & $-8,30$ \\
\hline Distrito Federal & 5,8 & 6,6 & 0,80 & 8,5 & 6,0 & $-2,50$ \\
\hline
\end{tabular}

Fonte: Autores/Dados extraídos da Pesquisa Nacional de Saúde (2013 e 2019).

A Tabela 2 mostra que houve aumento na proporção de pessoas, com 18 anos ou mais, que utilizaram medicamentos ou insulina para controle da diabetes em todas as UF, com variação de 48,1-93,7\%, entretanto, 22 UF (81,48\%) apresentaram redução na proporção de indivíduos que obteve algum medicamento por meio do programa "Farmácia Popular", com variação 
de 13,9-69,7\%. Nota-se que em todas as UF, da região norte, a proporção de pacientes que obteve alguma medicação pelo referido programa ficou abaixo de 50\% em 2019.

Tabela 2. Evolução da proporção de pessoas (\%) com 18 anos ou mais, que referem diagnóstico médico de diabetes e tomaram medicamento para controle da doença nas duas semanas anteriores à realização da pesquisa e da proporção de pessoas (\%) que tomaram algum medicamento para a diabetes obtido por meio do programa "Farmácia Popular", segundo as unidades federativas brasileiras, em 2013 e 2019.

\begin{tabular}{|c|c|c|c|c|c|c|}
\hline \multirow[t]{2}{*}{ Unidades federativas } & \multicolumn{3}{|c|}{$\begin{array}{l}\text { Pessoas que usaram medicamento ou } \\
\text { insulina nas duas últimas semanas (\%) }\end{array}$} & \multicolumn{3}{|c|}{$\begin{array}{c}\text { Pessoas que obtiveram algum medicamento no } \\
\text { programa "Farmácia Popular" }(\%)\end{array}$} \\
\hline & 2013 & 2019 & Diferença & 2013 & 2019 & Diferença \\
\hline \multicolumn{7}{|l|}{ Região Norte } \\
\hline Rondônia & 73,9 & 76,5 & 2,60 & 48,1 & 21,7 & $-26,40$ \\
\hline Acre & 48,1 & 84,0 & 35,90 & 63,2 & 24,7 & $-38,50$ \\
\hline Amazonas & 75,3 & 90,6 & 15,30 & 53,7 & 36,0 & $-17,70$ \\
\hline Roraima & 76,0 & 86,7 & 10,70 & 66,3 & 26,8 & $-39,50$ \\
\hline Pará & 79,5 & 88,6 & 9,10 & 41,4 & 41,1 & $-0,30$ \\
\hline Amapá & 68,7 & 82,1 & 13,40 & 45,0 & 13,9 & $-31,10$ \\
\hline Tocantins & 61,4 & 83,0 & 21,60 & 66,1 & 34,1 & $-32,00$ \\
\hline \multicolumn{7}{|l|}{ Região Nordeste } \\
\hline Maranhão & 81,9 & 90,0 & 8,10 & 50,9 & 31,4 & $-19,50$ \\
\hline Piauí & 69,9 & 84,1 & 14,20 & 45,2 & 42,6 & $-2,60$ \\
\hline Ceará & 72,6 & 90,3 & 17,70 & 64,5 & 33,7 & $-30,80$ \\
\hline Rio Grande do Norte & 78,7 & 84,1 & 5,40 & 57,8 & 52,1 & $-5,70$ \\
\hline Paraíba & 69,0 & 87,1 & 18,10 & 59,6 & 50,7 & $-8,90$ \\
\hline Pernambuco & 81,7 & 91,9 & 10,20 & 56,8 & 38,4 & $-18,40$ \\
\hline Alagoas & 73,5 & 93,7 & 20,20 & 58,0 & 41,9 & $-16,10$ \\
\hline Sergipe & 77,3 & 88,3 & 11,00 & 67,8 & 28,7 & $-39,10$ \\
\hline Bahia & 74,0 & 92,5 & 18,50 & 65,4 & 40,9 & $-24,50$ \\
\hline \multicolumn{7}{|l|}{ Região Sudeste } \\
\hline Minas Gerais & 80,2 & 86,9 & 6,70 & 61,9 & 63,1 & 1,20 \\
\hline Espírito Santo & 84,7 & 85,6 & 0,90 & 69,7 & 69,6 & $-0,10$ \\
\hline Rio de Janeiro & 87,9 & 91,8 & 3,90 & 44,6 & 58,2 & 13,60 \\
\hline São Paulo & 85,2 & 88,6 & 3,40 & 59,6 & 51,9 & $-7,70$ \\
\hline \multicolumn{7}{|l|}{ Região Sul } \\
\hline Paraná & 82,9 & 89,2 & 6,30 & 50,9 & 52,4 & 1,50 \\
\hline Santa Catarina & 74,2 & 86,7 & 12,50 & 61,1 & 59,6 & $-1,50$ \\
\hline Rio Grande do Sul & 72,5 & 85,3 & 12,80 & 54,5 & 64,7 & 10,20 \\
\hline \multicolumn{7}{|l|}{ Região Centro-Oeste } \\
\hline Mato Grosso do Sul & 75,3 & 90,8 & 15,50 & 62,0 & 36,1 & $-25,90$ \\
\hline Mato Grosso & 68,3 & 86,7 & 18,40 & 63,4 & 53,5 & $-9,90$ \\
\hline Goiás & 74,6 & 91,0 & 16,40 & 58,0 & 64,5 & 6,50 \\
\hline Distrito Federal & 85,9 & 91,1 & 5,20 & 65,0 & 58,7 & $-6,30$ \\
\hline
\end{tabular}


Verificou-se que houve aumento na proporção de pessoas, com 18 anos ou mais, que receberam assistência médica para diabetes no último ano em 22 UF (81,48\%), com variação de 53,2-87,3\%, acompanhada de redução na proporção de indivíduos que realizaram a última consulta em unidade básica de saúde em 12 UF (44,44\%), com variação de 29,4-68,8\% (Tabela 3).

Tabela 3. Evolução da proporção de pessoas (\%) com 18 anos ou mais, que referem diagnóstico médico de diabetes e receberam assistência médica para diabetes há menos de 1 ano e da proporção de pessoas (\%) que realizaram a última consulta em unidade básica de saúde, segundo as unidades federativas brasileiras, em 2013 e 2019.

\begin{tabular}{|c|c|c|c|c|c|c|}
\hline \multirow[t]{2}{*}{ Unidades federativas } & \multicolumn{3}{|c|}{$\begin{array}{c}\text { Pessoas que receberam assistência médica } \\
\text { para diabetes no último ano (\%) }\end{array}$} & \multicolumn{3}{|c|}{$\begin{array}{c}\text { Pessoas que realizaram a última consulta em } \\
\text { uma unidade básica de saúde }(\%)\end{array}$} \\
\hline & 2013 & 2019 & Diferença & 2013 & 2019 & Diferença \\
\hline \multicolumn{7}{|l|}{ Região Norte } \\
\hline Rondônia & 74,4 & 72,4 & $-2,00$ & 51,5 & 51,5 & 0,00 \\
\hline Acre & 65,4 & 75,0 & 9,60 & 66,5 & 68,8 & 2,30 \\
\hline Amazonas & 81,0 & 85,5 & 4,50 & 55,3 & 45,6 & $-9,70$ \\
\hline Roraima & 75,3 & 84,4 & 9,10 & 50,6 & 59,8 & 9,20 \\
\hline Pará & 78,9 & 83,0 & 4,10 & 44,5 & 60,9 & 16,40 \\
\hline Amapá & 80,7 & 81,9 & 1,20 & 55,8 & 48,5 & $-7,30$ \\
\hline Tocantins & 60,1 & 83,7 & 23,60 & 60,1 & 59,8 & $-0,30$ \\
\hline \multicolumn{7}{|l|}{ Região Nordeste } \\
\hline Maranhão & 72,3 & 80,3 & 8,00 & 37,6 & 44,7 & 7,10 \\
\hline Piauí & 68,6 & 86,6 & 18,00 & 45,0 & 51,0 & 6,00 \\
\hline Ceará & 74,8 & 77,2 & 2,40 & 64,4 & 56,4 & $-8,00$ \\
\hline Rio Grande do Norte & 68,4 & 87,3 & 18,90 & 42,8 & 46,3 & 3,50 \\
\hline Paraíba & 53,2 & 71,4 & 18,20 & 55,2 & 52,1 & $-3,10$ \\
\hline Pernambuco & 57,4 & 76,7 & 19,30 & 32,4 & 41,9 & 9,50 \\
\hline Alagoas & 74,5 & 83,0 & 8,50 & 53,2 & 56,6 & 3,40 \\
\hline Sergipe & 78,8 & 75,7 & $-3,10$ & 49,2 & 48,7 & $-0,50$ \\
\hline Bahia & 68,9 & 76,4 & 7,50 & 42,5 & 49,3 & 6,80 \\
\hline \multicolumn{7}{|l|}{ Região Sudeste } \\
\hline Minas Gerais & 80,4 & 78,5 & $-1,90$ & 53,6 & 46,0 & $-7,60$ \\
\hline Espírito Santo & 73,0 & 86,8 & 13,80 & 45,6 & 55,1 & 9,50 \\
\hline Rio de Janeiro & 76,8 & 82,1 & 5,30 & 29,4 & 35,5 & 6,10 \\
\hline São Paulo & 73,4 & 80,6 & 7,20 & 48,2 & 50,2 & 2,00 \\
\hline \multicolumn{7}{|l|}{ Região Sul } \\
\hline Paraná & 79,2 & 81,5 & 2,30 & 52,6 & 58,5 & 5,90 \\
\hline Santa Catarina & 66,4 & 76,6 & 10,20 & 67,8 & 66,8 & $-1,00$ \\
\hline Rio Grande do Sul & 69,5 & 67,6 & $-1,90$ & 38,8 & 46,8 & 8,00 \\
\hline \multicolumn{7}{|l|}{ Região Centro-Oeste } \\
\hline Mato Grosso do Sul & 71,4 & 78,9 & 7,50 & 58,6 & 53,3 & $-5,30$ \\
\hline Mato Grosso & 68,2 & 79,1 & 10,90 & 56,9 & 48,6 & $-8,30$ \\
\hline Goiás & 77,8 & 77,1 & $-0,70$ & 49,8 & 49,3 & $-0,50$ \\
\hline Distrito Federal & 77,5 & 81,4 & 3,90 & 48,0 & 47,1 & $-0,90$ \\
\hline
\end{tabular}


Fonte: Autores/Dados extraídos da Pesquisa Nacional de Saúde (2013 e 2019).

Notou-se que, na maioria das UF, houve diminuição na proporção de pessoas, com 18 anos ou mais, que conseguiu realizar todos os exames complementares solicitados $(n=17)$ e todas as consultas com médico especialista $(n=18)$ para as quais foram encaminhadas (Tabela 4). Em 2019, a proporção de pacientes realizou todos os exames complementares foi superior a 86\% em todas as UF, enquanto a execução de consultas com médico especialista teve uma proporção menor de consolidação, com as UF de Sergipe e Mato Grosso do Sul apresentando apenas cerca de 50\% das consultas realizadas.

Tabela 4. Evolução da proporção de pessoas (\%) com 18 anos ou mais, que referem diagnóstico médico de diabetes, e conseguiram realizar todos os exames complementares solicitados e da proporção de pessoas (\%) conseguiram realizar todas as consultas com médico especialista para as quais foram encaminhadas, segundo as unidades federativas brasileiras, em 2013 e 2019.

\begin{tabular}{|c|c|c|c|c|c|c|}
\hline \multirow{2}{*}{ Unidades federativas } & \multicolumn{3}{|c|}{$\begin{array}{l}\text { Pessoas que conseguiram realizar todos os } \\
\text { exames complementares solicitados (\%) }\end{array}$} & \multicolumn{3}{|c|}{$\begin{array}{l}\text { Pessoas que conseguiram realizar todas as } \\
\text { consultas com médico especialista (\%) }\end{array}$} \\
\hline & 2013 & 2019 & Diferença & 2013 & 2019 & Diferença \\
\hline \multicolumn{7}{|l|}{ Região Norte } \\
\hline Rondônia & 92,1 & 93,7 & 1,60 & 95,7 & 85,8 & $-9,90$ \\
\hline Acre & 89,3 & 93,6 & 4,30 & 71,5 & 81,8 & 10,30 \\
\hline Amazonas & 97,7 & 90,2 & $-7,50$ & 93,7 & 84,0 & $-9,70$ \\
\hline Roraima & 95,2 & 96,7 & 1,50 & 76,8 & 82,2 & 5,40 \\
\hline Pará & 75,4 & 91,9 & 16,50 & 84,2 & 81,4 & $-2,80$ \\
\hline Amapá & 94,8 & 92,6 & $-2,20$ & 85,1 & 90,4 & 5,30 \\
\hline Tocantins & 95,1 & 100,0 & 4,90 & 100,0 & 76,3 & $-23,70$ \\
\hline \multicolumn{7}{|l|}{ Região Nordeste } \\
\hline Maranhão & 92,6 & 91,2 & $-1,40$ & 84,1 & 64,1 & $-20,00$ \\
\hline Piauí & 95,8 & 91,7 & $-4,10$ & 93,6 & 94,8 & 1,20 \\
\hline Ceará & 92,7 & 90,8 & $-1,90$ & 80,9 & 75,8 & $-5,10$ \\
\hline Rio Grande do Norte & 93,1 & 93,3 & 0,20 & 70,2 & 76,6 & 6,40 \\
\hline Paraíba & 96,3 & 87,0 & $-9,30$ & 79,7 & 71,9 & $-7,80$ \\
\hline Pernambuco & 91,8 & 91,5 & $-0,30$ & 58,9 & 75,3 & 16,40 \\
\hline Alagoas & 86,5 & 91,5 & 5,00 & 76,9 & 73,9 & $-3,00$ \\
\hline Sergipe & 95,6 & 86,8 & $-8,80$ & 67,7 & 48,8 & $-18,90$ \\
\hline Bahia & 87,1 & 89,1 & 2,00 & 84,5 & 79,4 & $-5,10$ \\
\hline \multicolumn{7}{|l|}{ Região Sudeste } \\
\hline Minas Gerais & 98,1 & 95,9 & $-2,20$ & 74,4 & 73,0 & $-1,40$ \\
\hline Espírito Santo & 91,0 & 97,8 & 6,80 & 66,2 & 76,4 & 10,20 \\
\hline Rio de Janeiro & 93,7 & 92,3 & $-1,40$ & 79,5 & 84,0 & 4,50 \\
\hline São Paulo & 97,0 & 94,9 & $-2,10$ & 88,3 & 86,9 & $-1,40$ \\
\hline \multicolumn{7}{|l|}{ Região Sul } \\
\hline Paraná & 98,8 & 94,8 & $-4,00$ & 95,3 & 91,1 & $-4,20$ \\
\hline Santa Catarina & 100,0 & 94,5 & $-5,50$ & 89,5 & 77,3 & $-12,20$ \\
\hline Rio Grande do Sul & 98,9 & 93,0 & $-5,90$ & 72,0 & 95,0 & 23,00 \\
\hline
\end{tabular}




\begin{tabular}{|c|c|c|c|c|c|c|}
\hline Mato Grosso do Sul & 97,6 & 97,0 & $-0,60$ & 82,6 & 53,9 & $-28,70$ \\
\hline Mato Grosso & 98,2 & 99,0 & 0,80 & 91,0 & 83,3 & $-7,70$ \\
\hline Goiás & 98,0 & 93,6 & $-4,40$ & 94,9 & 88,7 & $-6,20$ \\
\hline Distrito Federal & 97,2 & 90,9 & $-6,30$ & 94,1 & 83,7 & $-10,40$ \\
\hline
\end{tabular}

Fonte: Autores/Dados extraídos da Pesquisa Nacional de Saúde (2013 e 2019).

Verificou-se que houve redução na proporção de pessoas, com 18 anos ou mais, que realizaram exame de vista há menos de 1 ano em 12 UF (44,44\%), com variação de 16,0-48,1\%, enquanto, a proporção de indivíduos que tiveram seus pés examinados há menos de 1 ano aumentou em 20 UF (74,07\%), com variação de 14,5-46,0\% (Tabela 5). A proporção de pacientes que realizaram exame de vista e tiveram seus pés examinado ficou abaixo de $50 \%$ em todas as UF.

Tabela 5. Evolução da proporção de pessoas (\%) com 18 anos ou mais, que referem diagnóstico médico de diabetes e realizaram exame de vista há menos de 1 ano e da proporção de pessoas (\%) que referem diagnóstico médico de diabetes e tiveram seus pés examinados há menos de 1 ano, segundo as unidades federativas brasileiras, em 2013 e 2019.

\begin{tabular}{|c|c|c|c|c|c|c|}
\hline \multirow[t]{2}{*}{ Unidades federativas } & \multicolumn{3}{|c|}{$\begin{array}{l}\text { Pessoas que realizaram exame de vista no } \\
\text { último ano }(\%)\end{array}$} & \multicolumn{3}{|c|}{$\begin{array}{c}\text { Pessoas que tiveram seus pés examinados } \\
\text { no último ano }(\%)\end{array}$} \\
\hline & 2013 & 2019 & Diferença & 2013 & 2019 & Diferença \\
\hline \multicolumn{7}{|l|}{ Região Norte } \\
\hline Rondônia & 16,0 & 25,1 & 9,10 & 20,4 & 19,9 & $-0,50$ \\
\hline Acre & 18,3 & 20,1 & 1,80 & 19,6 & 17,6 & $-2,00$ \\
\hline Amazonas & 44,9 & 45,6 & 0,70 & 35,3 & 43,3 & 8,00 \\
\hline Roraima & 39,8 & 36,2 & $-3,60$ & 46,0 & 30,6 & $-15,40$ \\
\hline Pará & 35,5 & 34,4 & $-1,10$ & 37,0 & 27,3 & $-9,70$ \\
\hline Amapá & 23,7 & 31,2 & 7,50 & 31,7 & 21,6 & $-10,10$ \\
\hline Tocantins & 28,7 & 23,3 & $-5,40$ & 19,8 & 31,7 & 11,90 \\
\hline \multicolumn{7}{|l|}{ Região Nordeste } \\
\hline Maranhão & 32,5 & 34,8 & 2,30 & 14,5 & 24,7 & 10,20 \\
\hline Piauí & 31,7 & 25,5 & $-6,20$ & 13,8 & 20,5 & 6,70 \\
\hline Ceará & 22,2 & 26,9 & 4,70 & 23,9 & 27,6 & 3,70 \\
\hline Rio Grande do Norte & 18,3 & 34,7 & 16,40 & 27,6 & 35,0 & 7,40 \\
\hline Paraíba & 25,9 & 31,4 & 5,50 & 17,6 & 28,3 & 10,70 \\
\hline Pernambuco & 27,3 & 38,5 & 11,20 & 18,2 & 27,2 & 9,00 \\
\hline Alagoas & 38,8 & 30,4 & $-8,40$ & 27,1 & 34,1 & 7,00 \\
\hline Sergipe & 33,1 & 23,8 & $-9,30$ & 18,0 & 21,4 & 3,40 \\
\hline Bahia & 25,6 & 33,8 & 8,20 & 28,0 & 34,4 & 6,40 \\
\hline \multicolumn{7}{|l|}{ Região Sudeste } \\
\hline Minas Gerais & 43,0 & 39,5 & $-3,50$ & 38,1 & 32,0 & $-6,10$ \\
\hline Espírito Santo & 38,8 & 42,3 & 3,50 & 22,2 & 31,3 & 9,10 \\
\hline Rio de Janeiro & 40,3 & 38,7 & $-1,60$ & 35,3 & 39,2 & 3,90 \\
\hline São Paulo & 40,1 & 42,1 & 2,00 & 32,6 & 32,8 & 0,20 \\
\hline
\end{tabular}


Região Sul

\begin{tabular}{lccccrr} 
Paraná & 29,6 & 35,4 & 5,80 & 26,0 & 31,7 & 5,70 \\
Santa Catarina & 42,5 & 38,8 & $-3,70$ & 21,5 & 35,1 & 13,60 \\
Rio Grande do Sul & 35,5 & 26,0 & $-9,50$ & 33,4 & 27,2 & $-6,20$ \\
Região Centro-Oeste & & & & & \\
Mato Grosso do Sul & 34,7 & 30,3 & $-4,40$ & 22,6 & 22,4 & $-0,20$ \\
Mato Grosso & 25,9 & 24,8 & $-1,10$ & 18,5 & 26,5 & 8,00 \\
Goiás & 23,3 & 39,0 & 15,70 & 14,5 & 28,9 & 14,40 \\
Distrito Federal & 42,3 & 48,1 & 5,80 & 26,1 & 35,2 & 9,10 \\
\hline
\end{tabular}

Fonte: Autores/Dados extraídos da Pesquisa Nacional de Saúde (2013 e 2019).

Conforme demonstrado na Tabela 6, observou-se que em 14 UF (51,85\%) observou-se aumento na proporção de pessoas, com 18 anos ou mais, que foram internadas por causa da diabetes ou de alguma complicação da doença, com variação de 4,7-31,4\%, enquanto a proporção de indivíduos com limitações nas atividades habituais diminuiu em 18 UF (66,67\%), com variação de $2,4-20,5 \%$.

Tabela 6. Evolução da proporção de pessoas (\%) com 18 anos ou mais, que referem diagnóstico médico de diabetes e foram internadas por causa da diabetes ou de alguma complicação da doença e da proporção de pessoas (\%) que possuem grau intenso ou muito intenso de limitações nas atividades habituais devido à diabetes ou de alguma complicação da doença, segundo as unidades federativas brasileiras, em 2013 e 2019.

\begin{tabular}{lrrrrrr}
\hline \multicolumn{1}{c}{ Unidades federativas } & $\begin{array}{c}\text { Pessoas que foram internadas por causa da } \\
\text { diabetes ou alguma complicação (\%) }\end{array}$ & \multicolumn{2}{c}{$\begin{array}{c}\text { Pessoas que possuem limitaços nas } \\
\text { atividades habituais (\%) }\end{array}$} \\
\hline Região Norte & 2013 & 2019 & Diferença & 2013 & 2019 & Diferença \\
Rondônia & & & & & & \\
Acre & 16,4 & 27,3 & 10,90 & 7,4 & 11,3 & 3,90 \\
Amazonas & 12,6 & 26,8 & 14,20 & 7,0 & 6,6 & $-0,40$ \\
Roraima & 7,4 & 14,8 & 7,40 & 3,3 & 6,5 & 3,20 \\
Pará & 31,4 & 18,5 & $-12,90$ & 20,5 & 2,8 & $-17,70$ \\
Amapá & 15,6 & 17,6 & 2,00 & 8,2 & 4,8 & $-3,40$ \\
Tocantins & 11,3 & 13,9 & 2,60 & 3,9 & 3,5 & $-0,40$ \\
Região Nordeste & 18,0 & 11,8 & $-6,20$ & 10,3 & 3,0 & $-7,30$ \\
Maranhão & & & & & & \\
Piaún & 9,5 & 24,1 & 14,60 & 11,0 & 9,3 & $-1,70$ \\
Ceará & 11,5 & 17,9 & 6,40 & 4,5 & 4,5 & 0,00 \\
Rio Grande do Norte & 15,9 & 13,7 & $-2,20$ & 5,5 & 5,2 & $-0,30$ \\
Paraíba & 7,9 & 17,1 & 9,20 & 7,8 & 2,9 & $-4,90$ \\
Pernambuco & 13,1 & 11,9 & $-1,20$ & 13,8 & 3,1 & $-10,70$ \\
Alagoas & 17,6 & 16,6 & $-1,00$ & 6,6 & 10,2 & 3,60 \\
Sergipe & 10,2 & 18,4 & 8,20 & 8,8 & 6,7 & $-2,10$ \\
Bahia & 16,3 & 12,4 & $-3,90$ & 7,6 & 9,1 & 1,50 \\
& 21,9 & 19,7 & $-2,20$ & 10,7 & 3,6 & $-7,10$
\end{tabular}




\begin{tabular}{lrrrrrr} 
Região Sudeste & & & & & & \\
Minas Gerais & 15,5 & 12,7 & $-2,80$ & 6,1 & 7,3 & 1,20 \\
Espírito Santo & 15,8 & 15,2 & $-0,60$ & 9,7 & 4,0 & $-5,70$ \\
Rio de Janeiro & 15,2 & 10,9 & $-4,30$ & 7,4 & 3,6 & $-3,80$ \\
São Paulo & 9,6 & 12,1 & 2,50 & 5,9 & 6,6 & 0,70 \\
Região Sul & & & & & & \\
Paraná & 11,1 & 19,6 & 8,50 & 2,4 & 6,3 & 3,90 \\
Santa Catarina & 16,1 & 12,2 & $-3,90$ & 18,2 & 8,5 & $-9,70$ \\
Rio Grande do Sul & 14,4 & 14,5 & 0,10 & 6,8 & 3,2 & $-3,60$ \\
Região Centro-Oeste & & & & & & \\
Mato Grosso do Sul & 17,3 & 17,4 & 0,10 & 5,6 & 4,8 & $-0,80$ \\
Mato Grosso & 4,7 & 16,6 & 11,90 & 4,8 & 10,3 & 5,50 \\
Goiás & 18,5 & 17,1 & $-1,40$ & 6,2 & 4,8 & $-1,40$ \\
Distrito Federal & 6,8 & 14,3 & 7,50 & 4,7 & 4,0 & $-0,70$ \\
\hline
\end{tabular}

Fonte: Autores/Dados extraídos da Pesquisa Nacional de Saúde (2013 e 2019).

\section{Discussão}

No presente estudo, constatou-se que a proporção de pessoas que referiram diagnóstico de diabetes aumentou em praticamente todas as UF brasileiras, evidenciando a importância do monitoramento contínuo das condições de saúde da população, visando nortear a elaboração de estratégias de prevenção e assistência à saúde do paciente com diabetes. Esse achado sugere que o enfrentamento da crescente prevalência da doença é uma preocupação para os serviços de saúde de todo o território nacional, independentemente do nível de desenvolvimento socioeconômico da região, e está de acordo com estudos que apontam que o diabetes é uma séria ameaça à saúde global que não respeita o status socioeconômico nem as fronteiras territoriais (International Diabetes Federation, 2019; Eqbal et al., 2020). Outros fatores têm sido associados à maior vulnerabilidade ao diabetes, como ser do sexo feminino, possuir idade avançada e baixo nível de escolaridade (Suwannaphant et al., 2017).

Adicionalmente, compreende-se que o aumento de indivíduos portadores de diabetes reflete o crescente problema da obesidade, sedentarismo e dieta inapropriada, evidenciando a necessidade de estratégias de conscientização sobre as complicações do diabetes, visando incorporar conhecimento, atitude e práticas saudáveis ao estilo de vida da população, prevenindo e melhorando o controle da doença (Castro et al., 2020; Sami et al., 2017; Leitner et al., 2017). Nesse sentido, é fundamental que os profissionais de saúde atuem, por meio de ações de educação em saúde, reforçando a importância da educação alimentar e da atividade física e estejam capacitados para auxiliar na introdução de hábitos compatíveis com a condição de vida do indivíduo (Corgozinho et al., 2020).

Concomitantemente ao aumento da proporção de pessoas que referiram diagnóstico de diabetes, observou-se declínio na proporção de indivíduos que nunca avaliaram a glicemia em todas as UF. Estes resultados podem estar relacionados, salientando que a realização de exames preventivos para detecção de alterações na glicemia, hemoglobina glicada e resistência à insulina pode contribuir para o diagnóstico precoce e enfrentamento do problema dos casos não diagnosticados da doença (Park, 2021). Estimativas globais apontam que, em 2019, a prevalência de diabetes não diagnosticado foi de 50,1\%, ou seja, 231,9 milhões dos 463 milhões de adultos que vivem com diabetes não sabem que têm a doença. O Brasil ocupa a sexta posição entre os países que possuem o maior número de adultos, com idade entre 20 e 79 anos, com diabetes não diagnosticado, apresentando 7,7 milhões de indivíduos, cerca de 46\% dos casos, em 2019 (International Diabetes Federation, 
2019). Essas estimativas evidenciam a necessidade urgente de aprimoramento das ações que contribuam para a detecção precoce da doença, uma vez que o diabetes não tratado pode ter severos efeitos negativos, como um maior risco complicações, aumento do uso dos serviços de saúde e custos relacionados (Cowie, 2019).

Outro aspecto de grande relevância para o controle do diabetes e de suas complicações é o acesso aos medicamentos de uso contínuo. Nesse contexto, destaca-se o Programa "Farmácia Popular", que foi criado com o objetivo de melhorar o acesso da população aos medicamentos considerados essenciais, reduzindo o impacto destes gastos no orçamento familiar, e constituindo-se em uma importante medida de saúde pública que pode contribuir para a melhoria da qualidade de vida dos pacientes com diabetes (Almeida et al., 2019). No presente estudo, observou-se que embora a proporção de pessoas que utilizaram medicamentos ou insulina para controle da diabetes tenha aumentado em todas as UF, houve redução na proporção dos que conseguiram o medicamento por meio do programa "Farmácia Popular" na maioria das UF, incluindo todas as das regiões Norte e Nordeste. É possível sugerir que estes achados estejam relacionados à aquisição dos medicamentos com custo integral, por parte dos indivíduos com melhor condição socioeconômica; que os medicamentos prescritos para o tratamento não estariam contemplados na lista do programa "Farmácia Popular"; e/ou que as farmácias da rede pública dos municípios dessas regiões estejam assegurando a disponibilidade dos medicamentos com maior regularidade (Costa et al., 2016). Estudos também ressaltam que para alcançar e manter o controle da doença em longo prazo, é necessária uma combinação de adequações no estilo de vida e tratamento farmacológico, evidenciando que os profissionais de saúde devem estar familiarizados com os diferentes tipos de medicamentos existentes para o tratamento do diabetes e selecionar os mais eficazes, seguros e melhor tolerados pelos pacientes (Tsukamoto et al., 2019; Marín-Peñalver et al., 2016).

Além da assistência farmacêutica, o acompanhamento médico periódico é crucial para avaliar a efetividade e/ou necessidade de adequação no plano de tratamento do paciente com diabetes. Nesse estudo, verificou-se aumento, na maioria das UF, na proporção de pessoas que receberam assistência médica para diabetes no último ano, ressaltando que em todas as UF a proporção de pessoas assistidas foi maior do que a de não assistidas. O aumento na proporção de pacientes diagnosticados também pode estar relacionado com o maior acesso aos serviços de saúde, evidenciando a necessidade de investimentos cada vez maiores para controlar a doença e suas complicações. Nesse contexto, salienta-se que além do impacto caracterizado pela mortalidade prematura e menor qualidade de vida, devido a complicações relacionadas ao diabetes, a doença também impõe um impacto econômico significativo sobre os países, sistemas de saúde e, quando os cuidados de saúde precisam ser financiados pelo próprio paciente, para os indivíduos com diabetes e suas famílias (Moreschi et al., 2020; American Diabetes Association, 2018).

Estimativas globais sugerem que, em 2019, os gastos na área da saúde relacionados ao diabetes chegaram a 760 bilhões de dólares, com tendência de crescimento para os próximos anos. O Brasil ocupa a terceira posição entre os países que possuem as maiores despesas na área da saúde devido ao diabetes, para assistência a pessoas com idade entre 20 e 79 anos, atingindo 52,3 bilhões de dólares, em 2019 (International Diabetes Federation, 2019). Simultaneamente ao aumento da proporção de pacientes que receberam assistência médica, notou-se que, em aproximadamente metade das UF, a maior proporção de pessoas não realizou sua última consulta em unidade básica de saúde. É possível sugerir que estes achados estejam relacionados à utilização de serviços privados; ampliação do uso de planos de saúde e convênios; e/ou ao aumento no referenciamento de pacientes para médicos especialistas. Nesse sentido, é importante considerar os possíveis fatores relacionados a este achado, pois a diferenciação no acesso aos serviços de saúde pode aumentar as iniquidades sociais diante de um sistema público de saúde constituído de modo universal e equânime (Cunha et al., 2019).

No contexto do acesso aos serviços de saúde, verificou-se que, em 2019, a maioria dos pacientes em todas as UF conseguiu realizar todos os exames complementares solicitados, enquanto a execução de consultas com médico especialista apresentou uma proporção menor de consolidação. Entretanto, deve-se alertar para o fato de que na maioria das UF houve 
diminuição na proporção de pessoas que conseguiu realizar todos os exames complementares solicitados e todas as consultas com médico especialista para as quais foram encaminhadas. Este achado está de acordo com estimativas que evidenciam a grande disparidade existente entre os países ao analisar as despesas de saúde atribuíveis ao diabetes por indivíduo, pois nota-se que, embora o Brasil seja um dos países com o maior número de pacientes com diabetes e que possuem as maiores despesas na área da saúde devido à doença, ao analisar a relação dos países com maiores despesas por indivíduo, observa-se o predomínio de países europeus que apresentam níveis elevados de qualidade de vida (International Diabetes Federation, 2019).

Estudos relatam a importância da ampliação da integração entre as unidades básicas e especializadas do Sistema Único de Saúde no atendimento ao paciente com diabetes, ressaltando que os pacientes que apresentam complicações da doença e que requerem necessidades de tratamento mais complexas apresentam pior qualidade de vida e são, geralmente, acompanhados pela Atenção Especializada (Corrêa et al., 2017; Brasil, 2013). Ademais, reforça-se que os exames laboratoriais complementares são fundamentais para o diagnóstico e acompanhamento da evolução dos casos de pacientes com diabetes, reforçando a importância de medidas que garantam não apenas a sua realização, mas a agilidade no processo de agendamento, execução e entrega dos resultados (Marinho et al., 2018).

No presente estudo, não foram investigados aspectos referentes a importantes variáveis que compõem a rede de assistência à saúde do paciente com diabetes, tais como o tempo para realização e entrega de resultados de exames complementares, tempo para agendamento de consultas com médicos especialistas, motivos para diminuição de obtenção de medicamentos por meio do Programa "Farmácia Popular" e de consultas com profissional das unidades básicas de saúde, o que pode ser considerado uma limitação da pesquisa e indica a necessidade de novos estudos complementares.

Em relação à realização de exames para prevenção e deteç̧ão precoce de complicações do diabetes, destaca-se, negativamente, que em todas as UF a maior parte dos pacientes com diabetes não realizou exame oftalmológico e nem tiveram seus pés examinados no último ano. A doença ocular diabética é uma das complicações mais temidas do diabetes, e compreende um grupo de doenças que incluem a retinopatia diabética, edema macular diabético, catarata e glaucoma, além de visão dupla e incapacidade de foco. Estimativas indicam que a prevalência global de retinopatia diabética é de 34,6\% e, em vários países, é uma das principais causas de cegueira e deficiência visual na população em idade produtiva, com consequências pessoais e socioeconômicas devastadoras, apesar de ser potencialmente evitável por meio de diagnóstico precoce e tratamento oportuno (Yau et al., 2012; Cavan et al., 2017; Pezzullo et al., 2018).

A deficiência no exame dos pés dos pacientes também traz grande preocupação devido a severidade das complicações e sequelas que podem ser evitadas a partir desse cuidado. A neuropatia periférica é a forma mais comum relacionada ao diabetes e sua prevalência relatada de varia de $16 \%$ até $87 \%$, afetando os nervos distais dos membros, principalmente os dos pés, alterando a função sensorial e causando dormência progressiva. Essas condições facilitam o desenvolvimento de úlceras resultantes de trauma externo, o chamado "pé diabético", cujas complicações estão relacionadas com lesões nos tecidos profundos associados a distúrbios neurológicos e doença vascular periférica nos membros inferiores (Sobhani et al., 2014). Úlceras nos pés e amputações são mais comuns em países de baixa e média renda, e estimativas globais sugerem que a cada 30 segundos um membro inferior, ou parte, é perdido devido à amputação como complicação do diabetes (Mishra et al., 2017; Amoah et al., 2018). Nesse contexto, estratégias preventivas importantes que podem reduzir o risco de amputação incluem o gerenciamento intensivo da glicemia; aumento da conscientização e do conhecimento dos profissionais de saúde sobre o manejo das complicações do pé diabético; realização regular de exames preventivos; e cuidados com os pés baseados na prevenção, educação e apoio por uma equipe multidisciplinar (Leal et al., 2020; International Diabetes Federation, 2019).

Como um possível reflexo da baixa taxa de realização de exames preventivos, no presente estudo, contatou-se um aumento na proporção de pessoas que foram internadas por causa da diabetes ou de alguma complicação da doença, na maioria das UF. Por outro lado, a proporção de indivíduos com limitações nas atividades habituais diminuiu na maioria das UF. Esses 
achados sugerem o elevado custo econômico que o tratamento e a reabilitação de pacientes têm gerado para o sistema de saúde, pois o diabetes e suas complicações, quando não adequadamente controlados, podem levar a internações hospitalares frequentes e morte prematura (International Diabetes Federation, 2019). Dados indicam que os custos diretos para o tratamento de pessoas com diabetes estão diretamente relacionados ao número de complicações presentes, sendo a média de gastos anuais com a saúde 20 vezes maior para pessoas com quatro ou mais complicações do que em pacientes sem complicações (Marcellusi et al., 2016). Adicionalmente, o impacto econômico da doença e suas complicações também se manifestam por meio dos custos gerados pela evasão de mão de obra, mortalidade, absenteísmo, e presenteísmo, destacando que a mortalidade contribui com 63,6\% dos custos indiretos em países de média renda e 90,6\% em países de baixa renda (Bommer et al., 2017).

Diante do exposto, compreende-se que o diabetes é um problema de saúde pública crescente que afeta todo o país, e que seu enfrentamento necessita de ações integradas e articuladas que envolvam não apenas a ampliação e o aprimoramento dos serviços de saúde, mas também a educação em saúde da população, visando a incorporação de hábitos de vida saudáveis e a conscientização sobre a magnitude do problema.

\section{Conclusão}

A prevalência da doença aumentou na ampla maioria das UF brasileiras e evidenciaram a necessidade de ampliação e aprimoramento das estratégias de educação em saúde, prevenção, assistência médica e farmacêutica ao paciente com diabetes, visando reduzir os agravos causados pela doença e suas complicações.

\section{Agradecimentos}

O presente trabalho foi realizado com apoio da Coordenação de Aperfeiçoamento de Pessoal de Nível Superior Brasil (CAPES) - Código de Financiamento 001.

\section{Referências}

Al-Lawati, J. A. (2017). Diabetes mellitus: A local and global public health emergency! Oman Medical Journal, 32(3), 177-179. https://doi.org/10.5001/omj.2017.34.

Almeida, A. T. C., Sá, E. B., Vieira, F. S., \& Benevides, R. P. (2019). Impacto do Programa Farmácia Popular do Brasil sobre a saúde de pacientes crônicos. Revista de Saúde Pública, 53, 20. http://dx.doi.org/10.11606/s1518-8787.2019053000733

American Diabetes Association. (2018). Economic costs of diabetes in the U.S. in 2017. Diabetes Care, 41(5), 917-928. https://doi.org/10.2337/dci18-0007

Amoah, V. M. K., Anokye, R., Acheampong, E., Dadson, H. R., Osei, M., \& Nadutey, A. (2018). The experiences of people with diabetes-related lower limb amputation at the Komfo Anokye Teaching Hospital (KATH) in Ghana. BMC Research Notes, 11(1), 66. https://doi.org/10.1186/s13104-018-3176-1

Bommer, C., Heesemann, E., Sagalova, V., Manne-Goehler, J., Atun, R., Bärnighausen, T., \& Vollmer, S. (2017). The global economic burden of diabetes in adults aged 20-79 years: a cost-of-illness study. The Lancet. Diabetes \& Endocrinology, 5(6), 423-430. https://doi.org/10.1016/S2213-8587(17)30097-9

Brasil. Ministério da Saúde. Estratégias para o cuidado da pessoa com doença crônica: diabetes mellitus. Brasília: Ministério da Saúde, 2013.

Brasil. Ministério da Saúde. Plano de reorganização da atenção à hipertensão arterial e ao diabetes mellitus: hipertensão arterial e diabetes mellitus. Brasília: Ministério da Saúde, 2001.

Brasil. Pesquisa nacional de saúde 2013: percepção do estado de saúde, estilos de vida e doenças crônicas: Brasil, grandes regiões e unidades da federação. Rio de Janeiro: Instituto Brasileiro de Geografia e Estatística, 2014.

Brasil. Pesquisa nacional de saúde 2019: percepção do estado de saúde, estilos de vida, doenças crônicas e saúde bucal: Brasil e grandes regiões. Rio de Janeiro: Instituto Brasileiro de Geografia e Estatística, 2020.

Cavan, D., Makaroff, L., Fernandes, J. R., Sylvanowicz, M., Ackland, P., Conlon, J., Chaney, D., Malhi, A., \& Barratt, J. (2017). The diabetic retinopathy barometer study: Global perspectives on access to and experiences of diabetic retinopathy screening and treatment. Diabetes Research and Clinical Practice, 129, 16-24. https://doi.org/10.1016/j.diabres.2017.03.023

Castro, I. B., Câmara, G. B., Pontes, J. F., Viana, D. L., Souza, R. P., Nobrega, E. D. S., Lira, R. B. B., \& Barbosa, L. S. L. T. (2020). Estratégias nutricionais no tratamento do diabetes mellitus: revisão bibliográfica. Research, Society and Development, 9, e133922193. 2020. DOI: http://dx.doi.org/10.33448/rsdv9i2.2193 
Corgozinho, M. L. M. V., Lovato, A. C., Martins, I. C. F., Mota, A. P. L., \& Mendes, A. C. R. (2020). Educação em diabetes e mudanças nos hábitos de vida. Research, Society and Development, 9, e175932566. https://doi.org/10.33448/rsd-v9i3.2566

Corrêa, K., Gouvêa, G. R., Silva, M. A. V., Possobon, R. F., Barbosa, L. F. L. N., Pereira, A. C., Miranda, L. G., \& Cortellazzi, K. L. (2017). Qualidade de vida e características dos pacientes diabéticos. Ciência \& Saúde Coletiva, 22(3), 921-930. https://doi.org/10.1590/1413-81232017223.24452015

Costa, K. S., Tavares, N. U. L., Mengue, S. S., Pereira, M. A., Malta, D. C., \& Silva Júnior, J. B. (2016). Obtenção de medicamentos para hipertensão e diabetes no Programa Farmácia Popular do Brasil: resultados da Pesquisa Nacional de Saúde, 2013. Epidemiologia e Serviços de Saúde, 25(1), 33-44. https://doi.org/10.5123/s1679-49742016000100004

Cowie, C. C. (2019). Diabetes diagnosis and control: Missed opportunities to improve health: The 2018 Kelly West Award Lecture. Diabetes Care, 42(6), 994-1004. https://doi.org/10.2337/dci18-0047

Cunha, C. L. F., Moreira, J. P. L., Oliveira, B. L. C. A., Bahia, L., \& Luiz, R. R. (2019). Planos privados de saúde e a saúde dos trabalhadores do Brasil. Ciência \& Saúde Coletiva, 24(5), 1959-1970. http://dx.doi.org/10.1590/1413-81232018245.20142017

DiMeglio, L. A., Evans-Molina, C., \& Oram, R. A. (2018). Type 1 diabetes. Lancet, 391(10138), 2449-2462. https://doi.org/10.1016/S0140-6736(18)31320-5

Eqbal, K., Alam, M. A., Quamri, M. A., Sofi, G., \& Bhat, M. D. A. (2020). Efficacy of Qurs-e-Gulnar in Ziabetus (type 2 Diabetes Mellitus): a single blind randomized controlled trial. Journal of Complementary \& Integrative Medicine, 18(1), 147-153. https://doi.org/10.1515/jcim-2020-0072

International Diabetes Federation. IDF Diabetes Atlas. (9th ed.). International Diabetes Federation, 2019.

Jing, X., Chen, J., Dong, Y., Han, D., Zhao, H., Wang, X., Gao, F., Li, C., Cui, Z., Liu, Y., \& Ma, J. (2018). Related factors of quality of life of type 2 diabetes patients: a systematic review and meta-analysis. Health and Quality of Life Outcomes, 16(1), 189. https://doi.org/10.1186/s12955-018-1021-9

Kao, K. T., \& Sabin, M. A. Type 2 diabetes mellitus in children and adolescents. Australian Family Physician, 45(6), $401-406$.

Leal, T. C., Budó, M. L. D., Schimith, M. D., \& Simon, B. S. (2020). Saberes e as práticas de pessoas com diabetes mellitus tipo 2 acerca do pé diabético. Research, Society and Development, 9, e618974511, 2020. http://dx.doi.org/10.33448/rsd-v9i7.4511

Leitner, D. R., Frühbeck, G., Yumuk, V., Schindler, K., Micic, D., Woodward, E., \& Toplak, H. (2017). Obesity and type 2 diabetes: Two diseases with a need for combined treatment strategies - EASO can lead the way. Obesity Facts, 10(5), 483-492. https://doi.org/10.1159/000480525

Leslie, R. D., Palmer, J., Schloot, N. C., \& Lernmark, A. (2016). Diabetes at the crossroads: relevance of disease classification to pathophysiology and treatment. Diabetologia, 59, 13-20. https://doi.org/10.1007/s00125-015-3789-z

Marcellusi, A., Viti, R., Sciattella, P., Aimaretti, G., Cosmo, S., Provenzano, V., Tonolo, G., \& Mennini, F. S. (2016). Economic aspects in the management of diabetes in Italy. BMJ Open Diabetes Research \& Care, 4(1), e000197. http://dx.doi.org/10.1136/bmjdrc-2016-000197

Marinho, N. B. P., Freitas, R. W. J. F., Lisboa, K. W. S. C., Alencar, A. M. P. G., Rebouças, V. C. F., \& Damasceno, M. M. C. (2018). Avaliação da satisfação de usuários de um serviço especializado em diabetes mellitus. Revista Brasileira de Enfermagem, 71(Suppl 1), 599-606. https://doi.org/10.1590/0034-71672017-0554.

Marín-Peñalver, J. J., Martín-Timón, I., Sevillano-Collantes, C., \& Del Cañizo-Gómez, F. J. (2016). Update on the treatment of type 2 diabetes mellitus. World Journal of Diabetes, 7(17), 354-395. https://dx.doi.org/10.4239/WJD.V7.I17.354

Mishra, S. C., Chhatbar, K. C., Kashikar, A., \& Mehndiratta, A. (2017). Diabetic foot. BMJ: British Medical Journal, 359, j5064. https://doi.org/10.1136/bmj.j5064

Moreschi, C., Rempel, C., Siqueira, D. F., Pissaia, L. F., Almeida, G., \& Bedin, B. B. (2020). Consequências do diabetes na qualidade de vida de usuários na ótica de profissionais de saúde. Research, Society and Development, 9, e801974818. http://dx.doi.org/10.33448/rsd-v9i7.4818

Papatheodorou, K., Banach, M., Bekiari, E., Rizzo, M., \& Edmonds, M. (2018). Complications of Diabetes 2017. Journal of Diabetes Research, 2018, 3086167. https://doi.org/10.1155/2018/3086167

Park, J. J. (2021). Epidemiology, pathophysiology, diagnosis and treatment of heart failure in diabetes. Diabetes \& Metabolism Journal, 45(2), 146-157. https://doi.org/10.4093/dmj.2020.0282

Pezzullo, L., Streatfeild, J., Simkiss, P., \& Shickle, D. (2018). The economic impact of sight loss and blindness in the UK adult population. BMC Health Services Research, 18(1), 63. https://doi.org/10.1186/s12913-018-2836-0

Sami, W., Ansari, T., Butt, N. S., \& Hamid, M. R. A. (2017). Effect of diet on type 2 diabetes mellitus: A review. International Journal of Health Sciences, $11(2), 65-71$.

Skyler, J. S., Bakris, G. L., Bonifacio, E., Darsow, T., Eckel, R. H., Groop, L., Groop, P. H., Handelsman, Y., Insel, R. A., Mathieu, C., McElvaine, A. T., Palmer, J. P., Pugliese, A., Schatz, D. A., Sosenko, J. M., Wilding, J. P., \& Ratner, R. E. (2017). Differentiation of diabetes by pathophysiology, natural history, and prognosis. Diabetes, 66(2), 241-255. https://doi.org/10.2337/db16-0806

Sobhani, S., Asayesh, H., Sharifi, F., Djalalinia, S., Baradaran, H. R., Arzaghi, S. M., Mansourian, M., Rezapoor, A., Ansari, H., Masoud, M. P., \& Qorbani, M. (2014). Prevalence of diabetic peripheral neuropathy in Iran: a systematic review and metaanalysis. Journal of Diabetes and Metabolic Disorders, 13(1), 97. https://doi.org/10.1186/s40200-014-0097-y

Suwannaphant, K., Laohasiriwong, W., Puttanapong, N., Saengsuwan, J., \& Phajan, T. (2017). Association between socioeconomic status and diabetes mellitus: The national socioeconomics survey, 2010 and 2012. Journal of Clinical and Diagnostic Research: JCDR, 11(7), LC18-LC22. https://doi.org/10.7860/JCDR/2017/28221.10286 
Research, Society and Development, v. 10, n. 5, e13510514776, 2021

(CC BY 4.0) | ISSN 2525-3409 | DOI: http://dx.doi.org/10.33448/rsd-v10i5.14776

Tsukamoto, K., Cnop, M., Mori, D., Kume, S., Anazawa, T., Doi, M., Chikazawa, K., \& Matsumaru, N. (2019). Future perspectives for the treatment of diabetes: Importance of a regulatory framework. Therapeutic Innovation \& Regulatory Science, 53(4), 535-541. https://doi.org/10.1177/2168479018795854

World Health Organization. Classification of diabetes mellitus. World Health Organization, 2019.

Yau, J. W., Rogers, S. L., Kawasaki, R., Lamoureux, E. L., Kowalski, J. W., Bek, T., Chen, S. J., Dekker, J. M., Fletcher, A., Grauslund, J., Haffner, S., Hamman, R. F., Ikram, M. K., Kayama, T., Klein, B. E., Klein, R., Krishnaiah, S., Mayurasakorn, K., O'Hare, J. P., Orchard, T. J., Porta, M., Rema, M., Roy, M. S., Sharma, T., Shaw, J., Taylor, H., Tielsch, J. M., Varma, R., Wang, J. J., Wang, N., West, S., Xu, L., Yasuda, M., Zhang, X., Mitchell, P., Wong, T. Y., \& Meta-Analysis for Eye Disease (META-EYE) Study Group. (2012). Global prevalence and major risk factors of diabetic retinopathy. Diabetes Care, 35(3), 556-564. https://doi.org/10.2337/dc11-1909 